\title{
A Worksite Self-management Program for Workers with Chronic Health Conditions Improves Worker Engagement and Retention, but not Workplace Function
}

\author{
William S. Shaw ${ }^{1}$ (D) $\cdot$ Robert K. McLellan ${ }^{2} \cdot$ Elyssa Besen $^{3} \cdot$ Sara Namazi $^{1} \cdot$ Michael K. Nicholas $^{4} \cdot$ Alicia G. Dugan $^{1}$. \\ Torill H. Tveito 5
}

Accepted: 26 April 2021 / Published online: 13 May 2021

(C) The Author(s) 2021

\begin{abstract}
Purpose An increasing number of workers in the US have chronic health conditions that limit their ability to work, and few worksite interventions have been tested to improve worker coping and problem solving at work. The purpose of this study was to evaluate a worksite-based health self-management program designed to improve workplace function among workers with chronic health conditions. Methods We conducted a randomized, controlled trial of a worksite self-management program ("Manage at Work") (clinicaltrials.gov \#NCT01978392) for workers with chronic health conditions (N=119; 82\% female, ages 20-69). Most workers were recruited from the health care or light manufacturing industry sectors. Workers attended a 5-session, facilitated psychoeducational program using concepts of health self-management, self-efficacy, ergonomics, and communication. Changes on outcomes of work engagement, work limitation, job satisfaction, work fatigue, work self-efficacy, days absent, and turnover intention at 6-month follow-up were compared to wait-list controls. Results The most prevalent chronic health conditions were musculoskeletal pain, headaches, vision problems, gastrointestinal disorders, respiratory disorders, and mental health disorders. The self-management program showed greater improvement in work engagement and turnover intent at 6-month follow-up, but there was no evidence of a parallel reduction in perceived work limitation. Trends for improved outcomes of work self-efficacy, job satisfaction, and work fatigue in the intervention group did not reach statistical significance in a group x time interaction test. Conclusions Offering a worksite self-management program to workers with chronic health conditions may be a feasible and beneficial strategy to engage and retain skilled workers who are risking disability.
\end{abstract}

Clinical trial registration: Clinicaltrials.gov \#NCT01978392.

Keywords Self-management · Worksite intervention · Chronic health conditions · Work engagement · Work limitation

William S. Shaw

wshaw@uchc.edu

Robert K. McLellan

Robert.k.mclellan@dartmouth.edu

Elyssa Besen

elyssa.saffrin@libertymutual.com

Sara Namazi

namazi@uchc.edu

Michael K. Nicholas

michael.nicholas@sydney.au.edu

Alicia G. Dugan

adugan@uchc.edu

Torill H. Tveito

torill.tveito@usn.no
1 University of Connecticut School of Medicine, 263 Farmington Ave., Farmington, CT 06030, USA

2 Geisel School of Medicine at Dartmouth, 1 Rope Ferry Drive, Hanover, NH 03755, USA

3 Liberty Mutual Insurance, 175 Berkeley Street, Boston, MA 02116, USA

4 University of Sydney at North Shore Hospital, St. Leonards, NSW 2065, Australia

5 University of South-Eastern Norway, Raveien 215, 3184 Borre, Norway 


\section{Introduction}

An important and growing source of job stress for many workers is the challenge of managing one or more chronic medical conditions at work. Forty percent of US workers report persistent or recurrent musculoskeletal pain or other chronic physical health conditions that are currently limiting their ability to work $[1,2]$. With the increased age and obesity of the US workforce, the prevalence of chronic diseases is likely to increase [3] and even younger workers are showing a higher prevalence of obesity and chronic health symptoms $[4,5]$. The most prevalent chronic conditions impacting working-age adults include low back pain, arthritis, migraine headaches, depression, diabetes, heart disease, and asthma [6-8].

Chronic conditions can lead to unpredictable and fluctuating symptoms, daily oscillations in work tolerance and fatigue, increased injury risk, and complex self-care or treatment regimens [9-11]. For workers with chronic conditions, conventional health and disability employer benefits may fail to satisfy day-to-day needs for job flexibility, leeway, and organizational support to cope effectively with periodic symptoms and task limitations. Over time, this can lead to increased job stress, dissatisfaction, turnover, and long-term disability [12-18]. Persistent or intermittent symptoms and fluctuating dysfunction can challenge conventional workplace approaches to disability management and accommodation [11,19-21].

While legislative mandates exist for employers to support job accommodation in principle, workers with chronic medical conditions still report problems communicating their needs effectively, making allowable work style and workstation adjustments, dealing with discomfort, overcoming stigma and discrimination, and keeping up with productivity expectations [22-24]. To overcome these problems, workers report a number of coping strategies: leveraging available job leeway and flexibility, careful planning and decision-making at work, obtaining sufficient job assistance and social support in and out of work, and communicating needs effectively and judiciously with peers and supervisors $[25,26]$. In addition to its toll on workers, chronic illness increases employer costs through reduced productivity, high turnover rates, absenteeism, and health care expense [27-30]. To reduce these costs, more proactive strategies are needed for employers to help workers with episodic symptoms.

Besides objective work outcomes such as sickness absence and temporary disability leave, subjective selfreport scales have been helpful to detect early perceptions of job-related limitations, disengagement, coping, and dissatisfaction among workers with chronic health conditions [15, 23, 29]. One hypothesis from this literature is that workers with chronic health conditions are unable to recover from a high daily level of exhaustion after working hours, and this ultimately leads to job burnout over time [23, 31]. Another theory is that workers slowly lose confidence in their abilities to perform routine tasks, to keep up with productivity demands, and to contribute meaningfully to their working teams [15, 30, 32]. These feelings may be associated with decreased personal self-efficacy and reduced confidence to manage pain or solve health-related challenges at work [32].

In the following study, we report the results of a randomized, controlled trial of a 5-session, worksite-based selfmanagement program designed to address functional problems of workers with chronic health conditions (the Manage at Work study) [33]. We hypothesized that an employersupported group intervention program adapted from principles of pain and illness self-management to the workplace context would improve work engagement and reduce work limitations.

\section{Method}

\section{Participants}

Participants were employees with chronic health conditions who expressed interest in a program designed to improve coping and function at work. Most were recruited from four worksites in the northeastern USA, including two large hospitals, a regional health care system, and a high-technology manufacturing firm. Participants were required to be fulltime workers ( $>20 \mathrm{~h}$ per week) and 18 years or older. Participants were required to have at least one chronic physical health condition ( $>6$ months) and most participants reported more than one chronic condition in a baseline survey (see "Results" sect. below). To avoid unnecessary health disclosure, participants were not required to provide medical information about diagnoses to qualify for the study. Reading and speaking in English was an inclusionary criterion. We excluded workers expecting to retire or change jobs within the next 12 months and workers who were unable to attend group workshops before work, after work, or during lunch breaks. Workers did not participate in the sessions during their standard, paid working hours.

\section{Procedure}

Detailed study procedures, including steps of the intervention design process and a detailed list of survey measures, are described in a published study protocol [33] and in a clinical registry entry (clinicaltrials.gov, \#NCT01978392). The study was publicized through posted flyers and email announcements sent to the entire workforce. Occupational 
health and safety staff also referred workers who described chronic health conditions at the time of a workplace injury or disability absence. Interested workers contacted the local onsite project coordinator who provided information about the study, answered questions, screened participants, obtained informed consent, and administered the baseline survey. The research office then provided randomization to the group intervention arm or to a wait-list control arm. A block randomization procedure was used (blocked in groups of 20) by the project statistician. After randomization, a local project coordinator contacted individuals in the treatment arm with a schedule for upcoming group intervention meetings. Control participants were placed on a waiting list for a one-day condensed workshop presentation of program materials one year later. Both control and intervention participants worked in the same occupational settings, so cross-contamination was a potential threat to internal validity, but information about study enrollment was kept confidential.

Research associates contacted participants in both the intervention and control groups 6 months after enrollment to complete a follow-up survey (20-30 min). Participants completed the baseline survey administered via an electronic computer tablet on-site in a private location. The 6-month follow-up surveys were completed on-line, with participants receiving an e-mail message prompt with an individual survey link and instructions on how to complete the survey. Participants in both the control and intervention groups received a $\$ 50$ check for completion of each survey. The baseline survey asked participants for demographic information as well as baseline measures of covariates and main outcomes. The follow-up survey contained a similar battery of measures. There was no substantial deviation from the original study protocol, but funding for the project ended prematurely with the unexpected closing of the research institute sponsor. This resulted in a smaller than expected sample size and the inability to collect 12-month follow-up survey data.

\section{Group Intervention}

The intervention program was developed using principles of health self-management programs but with messages tailored specifically to workplace challenges rather than lifestyle challenges at home. The goal was to improve personal perceptions of workability despite chronic health conditions and intermittent or episodic symptoms. The group workshop intervention content was developed based on qualitative studies [23] and review of existing self-management intervention elements [34]. The goal of the Manage at Work intervention was to provide coaching, education, and skill development to workers to help with self-management of chronic conditions in the workplace. The program was consistent with existing pain and illness self-management programs; however, the key messages and discussions were focused on overcoming workplace functional challenges. Workshop topics included coping, modifying work, communicating effectively, dealing with negative thoughts and emotions, and self-management of overall health and wellbeing. The sessions included presentations by the facilitator, group discussions, self-assessment activities, and brief homework assignments.

Five specially trained group facilitators led group management workshops. Group facilitators were licensed psychologists or clinical social workers, some of whom were providing services as part of the company's Employee Assistance Program. Group meetings were apportioned differently, with some groups opting for five 2-h sessions and others preferring ten 1 -h sessions or seven 1.5 -h sessions, depending on the schedules and availability of participants. Group sizes varied from 3 to 10. EAP professionals participating as group facilitators reported productive group discussions and high participation levels of study participants, and facilitators voiced no major feedback or concerns about program content and format to the research investigators.

\section{Measures}

\section{Primary Outcome Measures}

Two workplace-relevant self-report scales were chosen as primary outcome measures to reflect two different perspectives on workplace function. The Work Limitations Questionnaire (WLQ) [35] is a 25-item self-report questionnaire that assesses the degree to which working individuals are experiencing limitations on-the-job due to their health problems and health-related productivity loss. Respondents rate the level of difficulty or ability to perform specific job demands including time management, physical demands, mental-interpersonal demands, and output demands. The WLQ responses are on a 5-point scale from " 1 " (all of the time) to " 5 " (none of the time). The scale has good internal consistency [36] and has been validated against other health and disability constructs. Scores can be translated into a single Productivity Index score that estimates the percentage loss in work output due to health concerns. The Utrecht Work Engagement Scale (UWES) was chosen as a second primary outcome measure to provide an alternative perspective on functionality at work. The short-form of the UWES [37] is a 9-item self-report questionnaire designed to measure the degree to which employees have a sense of energetic connection with their work activities and see themselves as able to deal with the demands of their job. The UWES responses are on a 7-point scale from " 0 " (never) to "6" (always or every day). The UWES has good psychometric properties [38] and captures more holistic views of work related to vigor, dedication, and absorption. 


\section{Secondary Outcome Measures and Covariates}

To provide a more in-depth collection of work outcomes in this population, we added other self-report scales encompassing workplace fatigue (the Occupational Fatigue Exhaustion Recovery [OFER] scale [39, 40]), turnover intention [41], job satisfaction [42], a work self-efficacy measure combining items from the Pain Self-Efficacy Questionnaire [43] and Return-to-Work Self-Efficacy [RTWSE19] scale [44]. Details of these scales and their scoring and psychometric properties are included in the published study protocol [33].

\section{Data Analyses}

As a first step in the analyses, baseline demographic and health variables were compared between the intervention and control groups to test whether randomization had produced two equivalent groups. Any variables that showed statistically significant group differences were retained as covariates in subsequent analyses. The primary analytic strategy was to compare the intervention and control groups on changes in outcome measures (WLQ and UWES) at 6-month follow-up using a repeated measures general linear model, with group assignment being the between-subjects factor and time (baseline vs. follow-up) being the withinsubjects factor and including any necessary demographic covariates. In the repeated measures model, a statistically significant group $\mathrm{x}$ time interaction term $(\mathrm{p}<0.05)$ was evidence of different group outcomes. All analyses were conducted using IBM SPSS Statistics for Windows [45].

\section{Results}

A total of 119 participants ( 98 female, 20 male, 1 unspecified gender) provided informed consent and were randomized to the intervention or control arms of the study. The most common occupations were administrative assistant (19\%), manager/supervisor (17\%), data analyst or research assistant (13\%), medical assistant (12\%), medical technologist (9\%), nurse or nursing assistant (7\%), and coding or billing specialist (7\%). Other occupations included lab scientists, engineers, teachers, cashiers, assemblers, and counselors. The study population was comprised of mostly skilled workers in technical jobs with at least moderate levels of job security and opportunities for advancement.

Participants endorsed a median of 3 chronic health conditions (range 1-11). The most frequent chronic conditions were as follows: back or neck problems $(85 \%)$, hand/arm problems $(61 \%)$, leg or feet problems $(55 \%)$, migraine or severe headaches $(43 \%)$, vision problems $(31 \%)$, stomach or bowel disorders (24\%), asthma, bronchitis, or emphysema (22\%), mental health disorders (17\%), hearing problems (12\%), cardiovascular disease (5\%), severe skin disorders (4\%), and diabetes (4\%). Participants responded to either employer-based ( $92 \%$ ) or community-based (8\%) announcements about the study. The most prevalent comorbidity was to report a chronic musculoskeletal pain problem affecting more than one body part. Most participants $(83.2 \%)$ were working in a healthcare setting. Participants reported missing from 0 to 70 workdays $(\mathrm{M}=5.7$, $\mathrm{SD}=12.1)$ due to chronic health conditions over the previous six months.

Table 1 shows demographic characteristics by study groups, and these data reflect a mostly White, college educated, middle-aged cohort of workers with significant industry experience and job tenure. Despite random assignment to the intervention and control arms of the study, there was a trend for the control group to be older (mean age $=47.9$ versus 44.2 ) and there was a statistically significant group difference on job tenure, with the control group having more workers with $>5$ years $(61 \%$ versus $42 \%)$. Because these two variables have implications for both work and health outcome variables, they were retained as covariates in the subsequent multivariate group comparisons of study outcomes. There were no statistically significant differences or notable trends between the control and intervention groups on any other demographic variables.

Attendance records maintained by group facilitators reflected a moderate to high level of engagement in the intervention group, with $81 \%$ of participants completing at least half of the workplace self-management sessions. Subjective ratings of satisfaction with the intervention program were also high (mean overall satisfaction rating of 8.8 out of 10). Participants reported no adverse events related to study participation.

The means and standard deviations for baseline and follow-up measures are shown in Table 2. Of the original sample $(\mathrm{n}=119), 98$ participants $(82 \%)$ completed the 6-month survey. All tests of statistically significant changes in groups are controlled for the baseline covariates of age and job tenure. Both groups showed increasing work limitations over the 6-month period, and there was no significant group $x$ time interaction $(p>0.05)$ that would suggest the intervention program had attenuated this effect. On work engagement, however, the intervention group improved slightly, while the control group experienced a decline over the same 6-month period (interaction test, $p<0.05$ ). There was also a statistically significant improvement in turnover intent for the intervention group versus the control group (interaction test, $p<0.05$ ). Secondary measures of work selfefficacy, work fatigue, and job satisfaction showed a trend for the intervention group to improve in contrast with the control group, but these differences did not reach statistical significance. 
Table 1 Baseline demographic characteristics of study participants by randomized group assignment

\begin{tabular}{|c|c|c|c|c|c|}
\hline & All participants $(\mathrm{n}=119)$ & $\begin{array}{l}\text { Intervention group } \\
(\mathrm{n}=60)\end{array}$ & Control group $(\mathrm{n}=59)$ & $t$ or $\chi^{2}$ & $p$ \\
\hline & $\mathrm{M}(\mathrm{SD}) / \mathrm{n}(\%)$ & $\mathrm{M}(\mathrm{SD}) / \mathrm{n}(\%)$ & $\mathrm{M}(\mathrm{SD}) / \mathrm{n}(\%)$ & & \\
\hline Age & $46.0(12.7)$ & $44.2(12.0)$ & $47.9(13.2)$ & $t=1.624$ & 0.107 \\
\hline Chronic health conditions & $3.7(1.9)$ & $3.7(2.1)$ & $3.6(1.7)$ & $t=0.208$ & 0.836 \\
\hline Children/dependents at home & & & & $\chi^{2}=1.66$ & 0.799 \\
\hline Yes & $45(37.8)$ & $22(36.7)$ & $23(39.0)$ & & \\
\hline No & $74(62.2)$ & $38(63.3)$ & $38(64.4)$ & & \\
\hline Gender & & & & $\chi^{2}=3.16$ & 0.206 \\
\hline Male & $20(16.8)$ & $13(21.7)$ & $7(11.9)$ & & \\
\hline Female & $98(82.4)$ & $46(76.7)$ & $52(88.1)$ & & \\
\hline Not specified & $1(0.8)$ & $1(1.7)$ & $0(0.0)$ & & \\
\hline Race & & & & $\chi^{2}=0.17$ & 0.918 \\
\hline Asian & $2(1.7)$ & $1(1.7)$ & $1(1.7)$ & & \\
\hline Black & $7(5.9)$ & $3(5.0)$ & $4(6.8)$ & & \\
\hline White & $108(90.8)$ & $55(91.7)$ & $53(89.8)$ & & \\
\hline Not reported & $2(1.7)$ & $1(1.7)$ & $1(1.7)$ & & \\
\hline Ethnicity & & & & $\chi^{2}=0.00$ & 1.000 \\
\hline Hispanic & $4(3.4)$ & $2(3.3)$ & $2(3.3)$ & & \\
\hline Non-Hispanic & $112(94.1)$ & $56(93.3)$ & $56(94.9)$ & & \\
\hline Not reported & $3(2.5)$ & $2(3.3)$ & $1(1.7)$ & & \\
\hline Marital status & & & & $\chi^{2}=2.21$ & 0.531 \\
\hline Never married & $25(21.0)$ & $12(20.0)$ & $13(22.0)$ & & \\
\hline Married/partnered & $67(56.3)$ & $35(58.3)$ & $32(54.2)$ & & \\
\hline Divorced/separated & $25(21.0)$ & $13(21.7)$ & $12(20.3)$ & & \\
\hline Widowed & $2(1.7)$ & $0(0.0)$ & $2(3.4)$ & & \\
\hline Annual income & & & & $\chi^{2}=5.00$ & 0.840 \\
\hline$\$ 10,000-\$ 19,999$ & 3 & 1 & 2 & & \\
\hline$\$ 20,000-\$ 29,999$ & 14 & 8 & 6 & & \\
\hline$\$ 30,000-\$ 39,999$ & 27 & 12 & 15 & & \\
\hline$\$ 40,000-\$ 49,999$ & 27 & 15 & 12 & & \\
\hline$\$ 50,000-\$ 59,999$ & 11 & 7 & 4 & & \\
\hline$\$ 60,000-\$ 69,999$ & 10 & 5 & 5 & & \\
\hline$\$ 70,000-\$ 79,999$ & 13 & 5 & 8 & & \\
\hline$\$ 80,000-\$ 89,999$ & 5 & 2 & 3 & & \\
\hline$\$ 90,000-\$ 99,999$ & 4 & 1 & 3 & & \\
\hline$\$ 100,000$ or over & 4 & 3 & 1 & & \\
\hline (Missing) & 1 & 1 & 0 & & \\
\hline Highest education & & & & $\chi^{2}=3.69$ & 0.450 \\
\hline$<12$ years & $1(0.8)$ & $0(0.0)$ & $1(1.7)$ & & \\
\hline High school & $9(7.6)$ & $6(10.0)$ & $3(5.1)$ & & \\
\hline Some college & $54(45.4)$ & $28(46.7)$ & $26(44.1)$ & & \\
\hline Bachelor's degree & $27(22.7)$ & $15(25.0)$ & $12(20.3)$ & & \\
\hline Post-bachelor's & $28(23.5)$ & $11(18.3)$ & $17(28.8)$ & & \\
\hline With current employer & & & & $\chi^{2}=14.17$ & 0.015 \\
\hline 0-6 months & $8(6.7)$ & $4(6.7)$ & $4(6.8)$ & & \\
\hline $6-12$ months & $8(6.7)$ & $8(13.3)$ & $0(0.0)$ & & \\
\hline $1-2$ years & $8(6.7)$ & $6(10.0)$ & $2(3.4)$ & & \\
\hline $2-5$ years & $34(28.6)$ & $17(28.3)$ & $17(28.8)$ & & \\
\hline $5-10$ years & $29(24.4)$ & $9(15.0)$ & $20(33.9)$ & & \\
\hline$>10$ years & $32(26.9)$ & $16(26.7)$ & $16(27.1)$ & & \\
\hline
\end{tabular}


Table 1 (continued)

\begin{tabular}{|c|c|c|c|c|c|}
\hline & All participants $(\mathrm{n}=119)$ & $\begin{array}{l}\text { Intervention group } \\
(\mathrm{n}=60)\end{array}$ & Control group $(n=59)$ & $t$ or $\chi^{2}$ & $p$ \\
\hline & $\mathrm{M}(\mathrm{SD}) / \mathrm{n}(\%)$ & $\mathrm{M}(\mathrm{SD}) / \mathrm{n}(\%)$ & $\mathrm{M}(\mathrm{SD}) / \mathrm{n}(\%)$ & & \\
\hline With current industry & & & & $\chi^{2}=3.67$ & 0.599 \\
\hline $0-6$ months & $2(1.7)$ & $0(0.0)$ & $2(3.4)$ & & \\
\hline $6-12$ months & $5(4.2)$ & $4(6.7)$ & $1(1.7)$ & & \\
\hline $1-2$ years & $10(8.4)$ & $5(8.3)$ & $5(8.5)$ & & \\
\hline $2-5$ years & $24(20.2)$ & $14(23.3)$ & $10(16.9)$ & & \\
\hline $5-10$ years & $26(21.8)$ & $12(20.0)$ & $14(23.7)$ & & \\
\hline$>10$ years & $52(43.7)$ & $25(41.7)$ & $27(45.8)$ & & \\
\hline Working $>1$ employer & & & & $\chi^{2}=0.01$ & 0.973 \\
\hline No & $105(88.2)$ & $53(88.3)$ & $52(88.1)$ & & \\
\hline Yes & $14(11.8)$ & $7(11.7)$ & 7 (11.9) & & \\
\hline
\end{tabular}

Table 2 Changes in work outcomes by group assignment

\begin{tabular}{|c|c|c|c|c|c|c|c|c|}
\hline \multirow[t]{2}{*}{ Variable } & \multicolumn{3}{|c|}{ Intervention group $(n=47)^{\mathrm{a}}$} & \multicolumn{3}{|c|}{ Control group $(n=51)^{\mathrm{b}}$} & \multicolumn{2}{|c|}{$\begin{array}{l}\text { Group } \\
\mathrm{x} \text { time } \\
\text { interaction }^{\mathrm{c}}\end{array}$} \\
\hline & Baseline M (SD) & 6-month M (SD) & $\begin{array}{l}\text { Effect size } \\
\text { (Cohen's d) }\end{array}$ & Baseline M (SD) & 6-month M (SD) & $\begin{array}{l}\text { Effect size } \\
\text { (Cohen's d) }\end{array}$ & $F$ & $p$ \\
\hline \multicolumn{9}{|c|}{ Primary outcome measures } \\
\hline Work engagement & $4.53(0.99)$ & $4.79(1.36)$ & +0.22 & $5.10(1.01)$ & $4.68(1.32)$ & -0.36 & 7.88 & 0.006 \\
\hline Work limitation & $7.66(4.80)$ & $8.15(5.16)$ & +0.10 & $6.79(4.90)$ & $7.77(5.51)$ & +0.19 & 0.16 & 0.686 \\
\hline \multicolumn{9}{|c|}{ Secondary outcome measures } \\
\hline Job satisfaction & $6.62(1.98)$ & $6.43(2.27)$ & -0.09 & $6.80(1.59)$ & $6.40(2.17)$ & -0.21 & 0.20 & 0.660 \\
\hline Work fatigue & $2.88(1.14)$ & $2.67(1.29)$ & -0.17 & $2.89(1.34)$ & $2.80(1.32)$ & -0.07 & 0.45 & 0.505 \\
\hline Work self-efficacy & $3.97(0.97)$ & $4.12(1.13)$ & +0.14 & $4.05(1.10)$ & $4.01(1.20)$ & -0.03 & 1.23 & 0.271 \\
\hline Days lost to illness & $4.57(9.91)$ & $2.82(5.66)$ & -0.22 & $7.88(15.64)$ & $8.25(14.45)$ & +0.02 & 0.26 & 0.615 \\
\hline Turnover intent & $2.21(1.10)$ & $2.12(1.14)$ & -0.08 & $2.03(1.04)$ & $2.41(1.26)$ & +0.33 & 4.05 & 0.047 \\
\hline
\end{tabular}

${ }^{\mathrm{a}}$ Group sample size reflects 13 cases lost to follow-up

${ }^{\mathrm{b}}$ Group sample size reflects 8 cases lost to follow-up

${ }^{\mathrm{c}}$ Includes covariates of age and years job tenure with employer

On the 6-month recall of days lost to illness there was a marked reduction in the intervention group (from 4.6 to 2.8) versus the control group (from 7.9 to 8.3), but this difference in means was not statistically significant due to the positive skew of these data (i.e., only a few workers reported a high number of days lost to ill health). However, a post-hoc analysis of the number of workers reporting more than 10 days of lost time (McNemar test, $p<0.05$ ) showed a statistically significant worsening of sick days in the control group (those with $>10$ days increased from 5 to 10 individuals) but not in the intervention group (those with $>10$ days decreased from 4 individuals to 1 individual).

\section{Discussion}

This study presents a novel employer-sponsored strategy to improve the working conditions for employees with chronic health problems using a group psycho-educational format. The conceptual framework for the intervention was based around concepts of self-efficacy and health self-management, but targeted to address challenges of communication, pacing, problem-solving, and job modification unique to the workplace setting. This registered randomized clinical trial (Clinicaltrials.gov: NCT01978392) provided a strong methodological framework to evaluate benefits of the MANAGE AT WORK intervention 
program. Though the project was ended prematurely due to an unexpected closing of the sponsoring research institute, preliminary results indicate an improvement in workplace engagement while other health and work outcomes are approaching statistically significant gains when compared with a wait-list control group.

This study adds to the growing literature on the struggles of aging or ill workers to stay on the job despite recurrent or episodic symptoms, but workplace intervention strategies for this population have been sparse in the occupational rehabilitation literature [44]. While much of the existing research has focused on quantifying health effects on work productivity, other research has been helpful to illustrate the more subjective struggles of workers to maintain social relations, negotiate regarding accommodations, develop a realistic understanding of personal capabilities, and to demonstrate an appropriate level of assertiveness and knowledge to overcome organizational barriers [46, 47]. Employers may find it difficult to provide a supportive work environment for workers with chronic health conditions when symptoms are intermittent and when there may be no explicit request for accommodation. As reported in previous studies of chronic medical conditions among working populations [48], many participants reported multiple comorbid health conditions, often involving multiple body parts and systems. The most prevalent problems related to widespread musculoskeletal pain.

One broad indication from the study is the relative success of recruiting at-risk employees, making meetings convenient and safe for participants, fostering discussion, and encouraging workplace self-management strategies without any reported incidents of negative interactions or adversarial relations at work. Thus, it may be possible to expand on workers' efforts to obtain needed assistance and manage their workloads responsibly without disrupting employers and co-workers. One limitation of this approach is its reliance on workers to make or request adaptations in the workplace. The communication module of the training was built around the idea of promoting assertive communication about health, but this was balanced by the realities of working relationships, organizational culture, and workplace codes of conduct. Similarly, the job modification module was designed to make better use of existing leeway and flexibility using a problem-solving approach, while considering potential organizational or task-related barriers and constraints. Some workplace accommodation requests require more formal medico-legal processes, but the focus of the intervention was on informal types of job modification and assistance shown to be key for this working population with more episodic and transient problems [23].

The two primary outcome measures were work engagement and work limitations. Work engagement was the outcome measure that showed the greatest responsiveness to the intervention program. This construct was introduced in recent years to capture a more qualitative element of job satisfaction and is defined as ".... positive, fulfilling, workrelated state of mind that is characterized by vigor, dedication, and absorption" [38]. For workers with chronic or episodic health problems, negative feelings about work may take the form of burn-out, exhaustion, feeling less capable and isolated, and experiencing a general disengagement from work that is well captured by this psychological construct. Our self-management intervention may address these beliefs by identifying more options for positive change, finding ways to communicate needs effectively to managers and co-workers, and building a sense of mastery to overcome future challenges. As such, the intervention may have led workers to feel more interested and active in their day-today work and less absorbed by growing health problems and feelings of helplessness.

The other primary outcome measure for the study was the Work Limitations Questionnaire (WLQ). In contrast to work engagement, the WLQ did not show improvement in response to the intervention in our preliminary analyses. This measure has been very instrumental in research and evaluation to quantify the work productivity losses attributable to various medical conditions [9, 15, 35], but it has only begun to be used as an individual-level metric for evaluating intervention effectiveness. The intervention failed to improve participants' perceived ability to meet time pressures or perform physically demanding aspects of their work relative to the control group. One possible explanation is that the WLQ was not sufficiently responsive to short-term intervention, as the measure has shown statistically significant improvements in only one published trial (for depression) $[49,50]$. Another possibility is that workers with chronic health concerns are less concerned about their raw productivity output than about their psychological well-being. The uptake of future workplace interventions for workers with chronic health conditions may depend on showing improved well-being while also enhancing productivity.

An important practical implication of the study is that employers can provide health coaching or other educational or counseling programs to workers with chronic or episodic illness in the same vein as other types of workplace health promotion (e.g., smoking cessation or weight loss programs). In this example, groups were facilitated by Employee Assistance Program (EAP) counselors, who were specially trained to deliver the intervention program. An advantage of this approach was that EAP counselors had both a strong mental health training background but also understood issues of organizational culture, workplace relationships, and disability employer regulations. A second implication of our results is that workers did experience some increased attachment to their jobs as a product of a facilitated group intervention addressing 
health-related challenges. Providing more options for dealing with workplace challenges and a greater sense of mastery may improve the dedication and commitment of workers with chronic health conditions.

Our study is not without limitations. The premature cessation of funding on the project led to a smaller sample size (119 vs. 300$)$ and a reduced statistical power to show significant differences between groups. In terms of the intervention itself, an organizational component may have reinforced or strengthened the self-management and coping efforts of workers. Using cluster randomized designs may help to reduce the potential for cross-contamination between intervention and control groups in future studies. Most participants reported multiple chronic conditions, so the effect of overlapping comorbidities was difficult to assess. A shared effort that places equal responsibility on workers and supervisors to solve problems would place less onus on workers and more emphasis on organizational factors that can be changed.

Participating organizations may have been more apt than the average employer to allow workers to communicate with managers and peers about health concerns without any threat of retaliation or punitive treatment, though this is certainly an area of concern for more widespread implementation. The study sample included only a few occupational and industry groups, and effectiveness of the intervention may vary by occupation and industry. Future studies might combine this type of program with other organizational efforts (e.g., supervisor training, communications from management, ergonomic evaluations, peer coaches) to respond to the problem more completely. Another future direction would be to develop readiness assessment tools for employers to participate in such programs targeting workers with chronic health conditions.

In conclusion, this study demonstrates the initial feasibility of offering an employer-sponsored psycho-educational program to workers with chronic physical health conditions, and participation in this program resulted in improved levels of work engagement. Workers with chronic health conditions represent a fast-growing subset of the workforce in the US and elsewhere, and novel intervention strategies involving employer partnerships are sorely needed. We conclude that a group psycho-educational program is a feasible and effective way to improve worker engagement for those workers with chronic health conditions. Further efforts to develop and test employersponsored programs of this type should be pursued in different jurisdictions, contexts, and medical conditions to understand feasibility and effectiveness for more widespread dissemination.
Author Contributions All authors whose names appear on the submission made substantial contributions to the conception or design of the work or drafted the work or revised it critically for important intellectual content. All authors have approved the final version to be published and are accountable for all aspects of the work.

Funding This research was funded through the intramural research program of the Liberty Mutual Research Institute for Safety (Project \#LMRIS 11-08 awarded to WS Shaw).

Data Availability A deidentified dataset for research purposes is available from the authors.

\section{Declarations}

Conflict of interest All authors declare that they have no conflicts of interest.

Ethical Approval This research was reviewed and approved by the Institutional Review Board of the Liberty Mutual Research Institute for Safety.

Consent to Participate All procedures followed were in accordance with the ethical standards of the responsible committee on human experimentation (institutional and national) and with the Helsinki Declaration of 1975, as revised in 2000 (5). Informed consent was obtained from all patients for being included in the study.

Open Access This article is licensed under a Creative Commons Attribution 4.0 International License, which permits use, sharing, adaptation, distribution and reproduction in any medium or format, as long as you give appropriate credit to the original author(s) and the source, provide a link to the Creative Commons licence, and indicate if changes were made. The images or other third party material in this article are included in the article's Creative Commons licence, unless indicated otherwise in a credit line to the material. If material is not included in the article's Creative Commons licence and your intended use is not permitted by statutory regulation or exceeds the permitted use, you will need to obtain permission directly from the copyright holder. To view a copy of this licence, visit http://creativecommons.org/licenses/by/4.0/.

\section{References}

1. Burton WN, Pransky G, Conti DJ, Chen CY, Edington DW. The association of medical conditions and presenteeism. J Occup Environ Med. 2004;46(6):S38-S45.

2. Ricci JA, Stewart WF, Chee E, Leotta C, Foley K, Hochberg MC. Back pain exacerbations and lost productive time costs in United States workers. Spine. 2006;31(26):3052-3060.

3. Leeuwen MT, Blyth FM, March LM, Nicholas MK, Cousins MJ. Chronic pain and reduced work effectiveness: the hidden cost to Australian employers. Eur J Pain. 2006;10(2):161-166.

4. Lee H, Lee D, Guo G, Harris KM. Trends in body mass index in adolescence and young adulthood in the United States, 19522002. J Adolescent Health. 2011;49(6):601-608.

5. Ward BW, Schiller JS. Prevalence of multiple chronic conditions among US adults: estimates from the National Health Interview Survey, 2010. Prev Chronic Dis. 2013;10(10):E65.

6. Brault M, Americans with disabilities. Current population reports. Washington, DC: US Census Bureau; 2005. p. P70-P177. 
7. Brault M, Hootman J, Helmick C, Theis K, Armour B. Prevalence and most common causes of disability among adults-United States, 2005. MMWR. 2009;58(16):421-426.

8. Murray CJ, Atkinson C, Ghalla K, Birbeck G, Burstein R, Chou D, et al. The state of US health, 1990-2010: burden of diseases, injuries, and risk factors. JAMA. 2013;310(6):591-608.

9. Lerner D, Allaire SH, Reisine ST. Work disability resulting from chronic health conditions. J Occup Environ Med. 2005;47(3):253-264.

10. Palmer KT, Harris EC, Coggon D. Chronic health problems and risk of accidental injury in the workplace: a systematic literature review. Occup Environ Med. 2008;65(11):757-764.

11. Pransky G, Fassier J-B, Besen E, Blanck P, Ekberg K, Feuerstein $\mathrm{M}$, et al. Sustaining work participation across the life course: addressing chronic health conditions in research of disability management practices. J Occup Rehabil. 2016;26(4):465-479.

12. Munir F, Yarker J, Haslam C, Long H, Leka S, Griffiths A, Cox S. Work factors related to psychological and health-related distress among employees with chronic illnesses. J Occup Rehabil. 2007; 17:259-277.

13. Detaille S, Heerkens YF, Engels JA, van der Gulden JW, van Dijk FJ. Common prognostic factors of work disability among employees with a chronic somatic disease: a systematic review of cohort studies. Scan J Work Environ Health. 2009;35(4):261-281.

14. Gignac MAM, Lacaille D, Beaton DE, Backman CL, Cao X, Badley EM. Striking a balance: work-health-personal life conflict in women and men with arthritis and its association with work outcomes. J Occup Rehabil. 2014;24:573-584.

15. Lerner D, Henke RM. What does research tell us about depression, job performance, and work productivity? J Occup Environ Med. 2008;50(4):401-410.

16. Schultz AB, Edington DW. Employee health and presenteeism: a systematic review. J Occup Rehabil. 2007;17(3):547-579.

17. Wang X, Guo J, Zhang X, Qu Z, Tian D, Ma S. The effects of depression and chronic diseases on the work outcomes of employees: a prospective study in Northwest China. Pub Health. 2014;128(8):734-742.

18. Yamada K, Matsudaira K, Imano H, Kitamura A, Iso H. Influence of work-related psychosocial factors on the prevalence of chronic pain and quality of life in patients with chronic pain. BMJ Open. 2016;6(4):e010356.

19. Wynne-Jones G, Buck R, Porteous C, Cooper L, Button LA, Main CJ, Phillips CJ. What happens to work if you're unwell? Beliefs and attitudes of managers and employees with musculoskeletal pain in a public sector setting. J Occup Rehabil. 2011;21:31-42.

20. Gould-Werth A, Morrison K, Ben-Shalom Y. Employers' perspectives on accommodating and retaining employees with newly acquired disabilities: an exploratory study. J Occup Rehabil. 2018;287:611-633.

21. Gignac MAM, Bowring J, Jetha A, Beaton DE, Breslin FC, Franche R-L, Irvin E, Macdermid JC, Shaw WS, Smith PM, Thompson A, Tompa E, Van Eerd D, Sanders R. Disclosure, privacy, and workplace accommodation of episodic disabilities: organizational perspectives on disability communication-support processes to sustain employment. J Occup Rehabil. 2021;31:153.

22. McGonagle AK, Barnes-Farrell JL. Chronic illness in the workplace: stigma, identity threat, and strain. Stress Health. 2014;30(4):310-321.

23. Tveito TH, Shaw WS, Huang Y-H, Nicholas MK, Wagner G. Managing pain in the workplace: a focus group study of challenges, strategies, and what matters most to workers with low back pain. Disabil Rehabil. 2010;32(24):2035-2045.

24. Varekamp I, Van Dijk F. Workplace problems and solutions for employees with chronic diseases. Occup Med. 2010;60(4):287-293.
25. Blyth FM, March LM, Nicholas MK, Cousins MJ. Selfmanagement of chronic pain: a population-based study. Pain. 2005;113(3):285-292.

26. Shaw WS, Feuerstein M, Haufler AJ, Berkowitz SM, Lopez MS. Working with low back pain: problem-solving orientation and function. Pain. 2001;93(2):129-137.

27. Burton WN, Chen C-Y, Schultz AB, Conti DJ, Pransky G, Edington DW. Worker productivity loss associated with arthritis. Dis Manage. 2006;9(3):131-143.

28. Collins JJ, Baase CM, Sharda CE, Ozminkowski RJ, Nicholson $\mathrm{S}$, Billotti GM, et al. The assessment of chronic health conditions on work performance, absence, and total economic impact for employers. J Occup Environ Med. 2005;47(6):547-557.

29. Goetzel RZ, Long SR, Ozminkowski RJ, Hawkins K, Wang S, Lynch W. Health, absence, disability, and presenteeism cost estimates of certain physical and mental health conditions affecting U.S. employers. J Occup Environ Med. 2004;46(4):398-412.

30. Koopmanschap M, Burdorf A, Lötters F. Work absenteeism and productivity loss at work. In: Loisel P, Anema JR, editors. Handbook of work disability. New York: Springer; 2013. pp. 31-41.

31. Boelhouwer IG, Vermeer W, van Vuuren T. Work ability, burnout complaints, and work engagement among employees with chronic diseases: job resources as targets for intervention. Front Psychol. 2020;11:1805.

32. Shaw WS, Huang Y. Concerns and expectations about returning to work with low back pain: identifying themes from focus groups and semi-structured interviews. J Occup Rehabil. 2005;27(21):1269-1281.

33. Shaw WS, Besen E, Pransky G, Boot CR, Nicholas MK, McLellan RK, et al. Manage at Work: a randomized, controlled trial of a self-management group intervention to overcome workplace challenges associated with chronic physical health conditions. BMC Public Health. 2014;14(1):515-525.

34. Shaw WS, Tveito TH, Geehern-Lavoie M, Huang Y-H, Nicholas MK, Reme SE, Pransky G. Adapting principles of chronic pain self-management to the workplace. Disabil Rehabil. 2012;34(8):694-703.

35. Lerner D, Amick BCI, Lee JC, Rooney T, Rogers WH, Chang H, Berndt ER. Relationship of employee-reported work limitations to work productivity. Med Care. 2003;41(5):649-659.

36. Lerner D, Chang H, Rogers WH, Benson C, Schein J, Allaire S. A method for computing the impact of health problems on at-work performance and productivity from available health data. J Occup Environ Med. 2009;51(5):515-524.

37. Demourati E, Bakker AB, De Jonge J, Janssen PP, Schaufeli WB. Burnout and engagement at work as a function of demands and control. Scan J Work Environ Health. 2001;17(4):279-286.

38. Schaufeli WB, Bakker AB, Salanova M. The measurement of work engagement with a short questionnaire: a cross-national study. Educ Psychol Meas. 2006;66(4):701-716.

39. Winwood PC, Winefield AH, Dawson D, Lushington K. Development and validation of a scale to measure work-related fatigue and recovery: the Occupational Fatigue Exhaustion/Recovery Scale (OFER). J Occup Environ Med. 2005;47(6):594-606.

40. Winwood PC, Lushginton K, Winefield AH. Further development and validation of the Occupational Fatigue Exhaustion Recovery (OFER) scale. J Occup Environ Med. 2006;48(4):381-389.

41. Kelloway EK, Gottlieb BH, Barham L. The source, nature, and direction of work and family conflict: a longitudinal investigation. J Occup Health Psychol. 1999;4(4):337.

42. Nagy MS. Using a single-item approach to measure facet job satisfaction. J Occup Organ Psychol. 2002;75(1):77-86.

43. Nicholas MK. The pain self-efficacy questionnaire: taking pain into account. Eur J Pain. 2007;11(2):153-163.

44. Shaw WS, Reme SE, Linton SJ, Huang Y-H, Pransky G. $3^{\text {rd }}$ place, PREMUS best paper competition: development of the 
return-to-work self-efficacy (RTWSE-19) questionnaire: psychometric properties and predictive validity. Scand J Work Environ Health. 2011;37(2):109-119.

45. Corp IBM. IBM SPSS Statistics for Windows, Version 25.0. Armonk, NY: IBM Corp; 2016.

46. Bosma AR, Boot CRL, Schaafsma FG, Kok G, Anema JR. Development of an intervention to create a supportive work environment for employees with chronic conditions: an intervention mapping approach. J Occup Rehabil. 2020;30:624.

47. Varekamp I, Heutink A, Landman S, Koning CEM, De Vries G, van Dijk FJH. Facilitating empowerment in employees with chronic disease: Qualitative analysis of the process of change. J Occup Rehabil. 2009;19:398-408.

48. Mayer TG, Towns BL, Neblett R, Theodore BR, Gatchel RJ. Chronic widespread pain in patients with occupational spinal disorders: prevalence, psychiatric comorbidity, and association with outcomes. Spine (Phila Pa 1976). 2008;33(17):1889-1897.

49. Lerner D, Adler D, Hermann RC, Chang H, Ludman EJ, Greenhill A, et al. Impact of a work-focused intervention on the productivity and symptoms of employees with depression. J Occup Environ Med. 2012;54(2):128-135.

50. Lerner D, Adler D, Rogers WH, Chang H, Greenhill A, Cymerman E, Azocar F. A randomized clinical trial of a telephone depression intervention to reduce employee presenteeism and absenteeism. Psychiatr Serv. 2015;66(6):570-577.

Publisher's Note Springer Nature remains neutral with regard to jurisdictional claims in published maps and institutional affiliations. 